\title{
Is Virtual Reality (VR) Becoming an Effective Application for the Market Opportunity in Health Care, Manufacturing, and Entertainment Industry?
}

\author{
Kay Kim \\ Fitchburg State University, Fitchburg MA USA
}

URL:http://dx.doi.org/10.19044/esj.2016.v12n9p14

\begin{abstract}
The purpose of this paper is to analyze how virtual reality technology has been blossoming and applying in the past few years to quickly become the next big new products development in high technology industry. Thanks to this technology being backed by major tech and manufacturing firms, such as Facebook, Ford, and Sony, it has shown much promise in impacting our lives by bringing something completely new to our experience of processing data. There are three sectors in particular where it benefits most; healthcare, manufacturing, and entertainment. The paper explains in detail how these areas are positively impacted and applied.
\end{abstract}

Keywords: Virtual reality, VR, technology, OcculusRift

\section{Introduction}

Virtual Reality (VR) brings a new way of analyzing and viewing data and video content. Through the use of a headset it is the only medium people can use to recreate real life. We will be able to communicate with the world around us in a controlled and realistic way. VR has been used by very few organizations within the past decade, who were able to afford its large cost. For example NASA has utilized this VR technology as part of their astronauts training in order to simulate tasks they must complete at the International Space Station.

Starting this year, 2016 organizations such as Facebook and Sony are investing billions of dollars in developing this product for businesses small and large. However, they plan to introduce it at an affordable price point so that it can be adopted by the general public.

This is an exciting time, when a new industry is blossoming right in front of our eyes. It is predicted that the VR industry will become a $\$ 140$ billion business and with it bring about a lot of unique and interesting ideas 
and concepts that will find ways to improve our lives for the better. VR will offer us a new way to disseminate information.

The quote from the movie Matrix may be a concept that will reign true in the near future. "What is real? How do you define real? If you're talking about what you can hear, what you can smell, taste and feel, then real is simply electrical signals interpreted by your brain." [9]

\section{Literature Reviews and Methods}

\section{Virtual Reality Application}

Virtual reality is generally understood by industry as a future technology that makes users feel in a virtual environment (VE) by using computer hardware and software. It was typically understood as a digitally created room which humans could access by donning special designated computer equipment. It make people to handle his/her information more easily in their virtual space.

VR utilizes that users a different way to see and experience information, one that is dynamic and immediate constantly. For example, in a computer game, user' joystick motions (behaviors) are tracked and the objects in the game are moved according to the joystick movements. In the same way a simulated, three-dimensional world is created around the user in which he/she could interact with objects, people, and environments. Generally, three-dimensional life-sized images with support of audio devices are introduced around the user and the perspective is adjusted in accordance with the user input (head or eye movements). Many devices along with the computers are used to create a virtual environment in this technology.

Under these above basic concept, VR technology has been applied and impacted on in many different industries. However, in this paper, the research has focused on the three most significant business trends in the areas of Health Care, Manufacturing, and Entertainment. These industries has been seen an entry of new and improved ways to approach not just services and production but also developing experience through virtual training.

For example in the area of HealthCare doctors can utilize VR business application to train in a lifelike operating room environment without the need of test patients or dummies.

It could be done from anywhere in the world, whether it be at the University lab or in their living rooms. Entertainment in the form of movies and videogames creates realistic environments. It heightens audience entertainment pleasure with a sense of realism that current 3-D technology cannot come close to replicating. To test of these circumstances, the method has implemented a qualitative literatures, and product case testing in these three industries introduced. 


\section{Virtual Reality used in HealthCare?}

VR technology has been making considerable progress in areas of healthcare. There are new and innovative ideas that will propel this industry to benefit from advancements in this technology. VR in healthcare will allow patients to be completely immersed in a computer generated a world. In this safe environment they will be able to undergo therapy. Skip Rizzo, a clinical psychologist and associate director for medical virtual reality at the University of Southern California's Institute for Creative Technologies, considers it a perfect pairing between VR and healthcare [10].

Rizzo is using Facebooks Oculus Rift [Figure 1] [5] for his virtual reality therapy to treat post-traumatic stress disorder. Rizzo is using this technology to help treat soldiers with PTSD. Prior to VR, soldiers in a physician's office would be instructed to close their eyes and envision those things or locations that they feared most. VR is enhancing their exposure in a controlled matter, with environments created in a virtual environment and putting ex-soldiers in situations where they are slowly exposed to events or locations that triggers their PTSD.

Figure 1. Leather, A (2014, April 14th). Para 1. Why Oculus Rift And Virtual Reality Could Be Huge And Not Just For Gamers. Retrieved from http://www.forbes.com/sites/antonyleather/2014/04/14/why-oculus-riftand-virtual-reality-could-be-huge-and-not-just-for-gamers/\#212ca8df651f

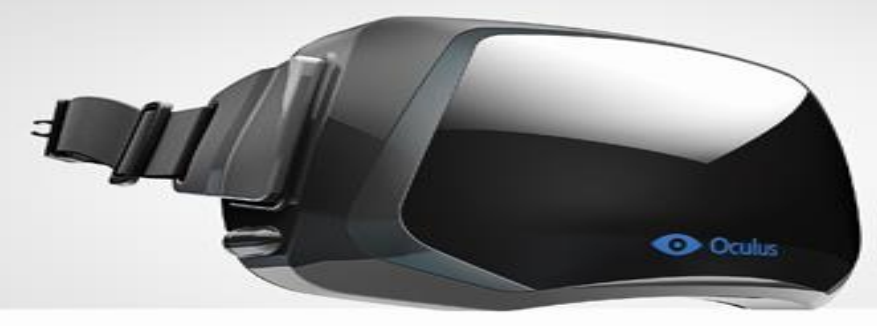

Doctors have found that facing those frightening moments will help an individual's better control PTSD symptoms and stop severe episodes before they happen. Rizzo has seen a lot of improvement in his patients PTSD symptoms after undergoing VR therapy. For example, Iraq war veterans are placed in realistic war torn virtual maps so that they can experience the horrors of war in a safe virtual environment. Situations like these help vets slowly improve and face their PTSD head on in the stage which a doctor can control.

Immersive experiences such as the exposure therapy used by PTSD patients can also be used for individuals who are fairly healthy and do not 
suffer from any mental disorders. Families or caregivers can use these devices to give them first-hand account of what their friend or loved one experiences. It helps instill a level of empathy in them and allows them to understand what others are experiencing.

A health care technology company called Viscira created a VR software for Facebook's Oculus Rift that stimulates schizophrenia in a healthy user. Individuals that have used it, have felt the emotional pain that those that have the disorder experience every day. The goal from that project is to create awareness and improve the level of support users with schizophrenia will receive. Viscira and organizations like that are helping to change people's behaviors toward illness in order for those individuals to work on improving the level of care. Other areas of healthcare that can also benefit, is meditation which can improve mental health, this concept was discussed by Senson [8].

Virtual locals can be created; for example, appearing on top of a mountain peak at looking down below at a beautiful landscape or overlooking a tranquil ocean from a sunny beach. These elements can deepen our meditative state and cause an enhancement in its relaxation benefits. This type of destressing technology can be used by anyone but many doctors want to introduce it to patients before they prepare for surgery. This technology that alleviate stress and ease tensions a patient will have prior to surgery.

It can also be used after surgery to help alleviate pain and make it a bit easier for patients to recover from their injuries in the hospital after a surgery. Studies have shown that patients who are happier and under less stress tend to heal faster.

\section{Virtual Reality (VR) in the Manufacturing Industry}

The automobile industry is implementing new and exciting ways of using VR technology to increase the efficiency in how it designs cars. Engineers at Ford have been experimenting with VR technology. According to Carr, [1] they have shifted focus to Facebook's Oculus Rift because of its cost efficiency and portability.

Car engineers can put on the Oculus rift head mounted device and find themselves inside the cockpit of a car they helped design. Using the devices motion capture cameras, users can turn their head and peak around corners just as they would in real life.

The software tracks a user's position so that when you move in the physical world that a person's viewpoint will change in the digital world. Auto designers can use this system to sit at the driver's seat, for example, and look out beyond the driver's window as if they were really driving. Designers can look at their vehicles with greater detail and study the internal fabrics and colors they have chosen. Designers can take it a step further and 
control the time of a day that they are in the car or outside the car. This will give them with the unique perspective of seeing how different colors and fabrics appear at various times of the day. Sunlight when bouncing off surfaces can alter its color.

Ford Motor Company has been at the forefront in adapting VR technology, which it began using in 1999. This was five years before Facebook purchased Oculus VR for $\$ 2$ billion in 2014. The purchase by Facebook highlighted VR into the global spotlight. However, VR has changed Ford's design approach for years. One big change VR has brought to Ford according to Gaudiosi [4]; is that Ford creates fewer physical models and has instead replaced that with a significantly lower cost virtual models.

Ford acknowledges that current consumer-level VR is not as sophisticated and doesn't have the high level fidelity of Ford's own FIVE technology, which stands for "Ford Immersive Vehicle Environment." Ford's tech allows engineers to view details of a car's upholstery or exterior groove lines within a half-millimeter. Ford is still excited because Facebook's platform brings a more user friendly format for thousands of developers to create applications for; furthermore its headset are more light weight and portable in comparison to what Ford uses.

Ford expects products such as Oculus to develop and become more sophisticated beyond its use as an entertainment vehicle. It sees it growing to purposes so that it can benefit professional users in a manufacturing capacity. Ford is very excited to embrace this technology in its early stages and see it evolve.

\section{Virtual Reality in Entertainment Industry}

Virtual reality will have its greatest impact in the general consumer market is in the area of entertainment VR has been referenced for years in pop cultural in films and T.V. shows However experts agree that this form of entertainment medium could have a deep and profound effect in the near future, Darell [2]. Organizations such as Facebook investing billions in VR and huge organizations such as Google are introducing their own iterations of VR tech [3].

Sony introducing their own VR platform under their PlayStation brand called "PlayStation VR." [Figure 2][6].

Figure 2. Maurizio, P. (2015, November 28). Nokia's Ozo VR Camera Marks a Rebirth for the Phone Giant. Retrieved from http://www.wired.com/2015/11/nokia-ozo-vr-camera/ 


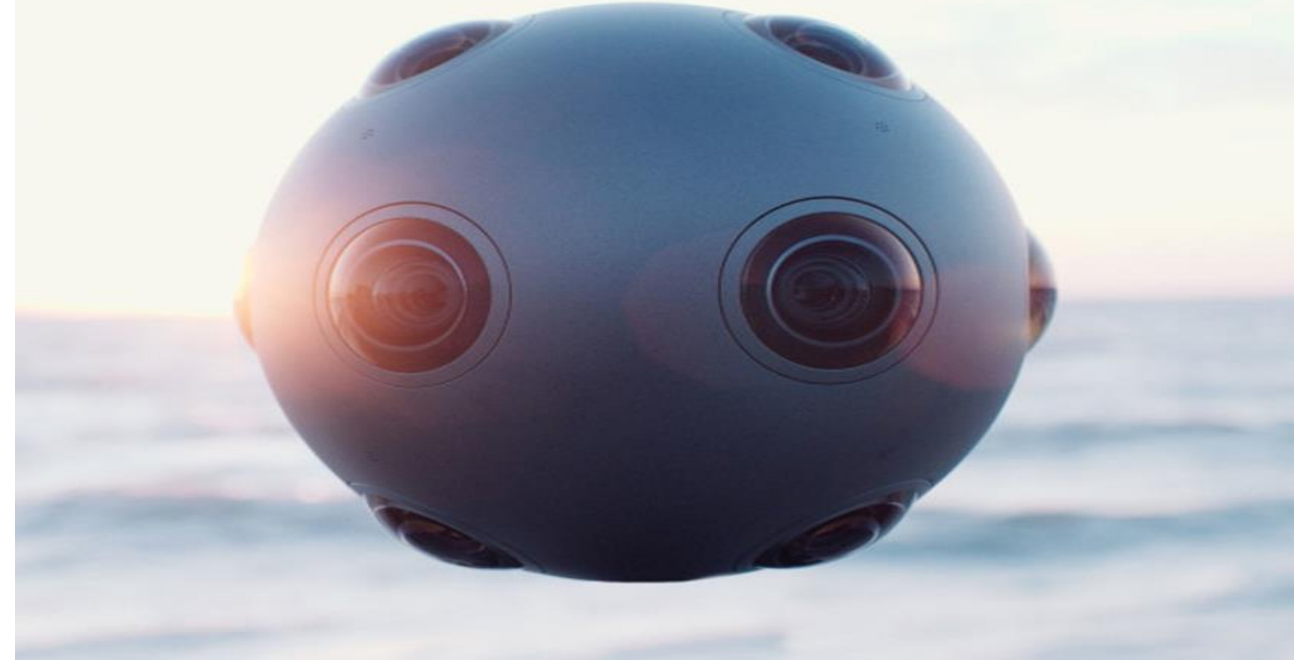

Sony is in an especially great position because they already have tens of millions of potential users that can enjoy the VR experience. Anyone of 35 million PlayStation 4 gaming console users are a potential customer. Within the next few years, Sony is on the pace of selling 140 million PlayStation units [Figure 3] [7].

Figure 3. Shanklin, W. (2016, January 11). PlayStation VR hands-on: A more mid-ranged alternative to the Rift and Vive. Retrieved from http://www.gizmag.com/playstation-vr-review-hands-on-2016/41286/

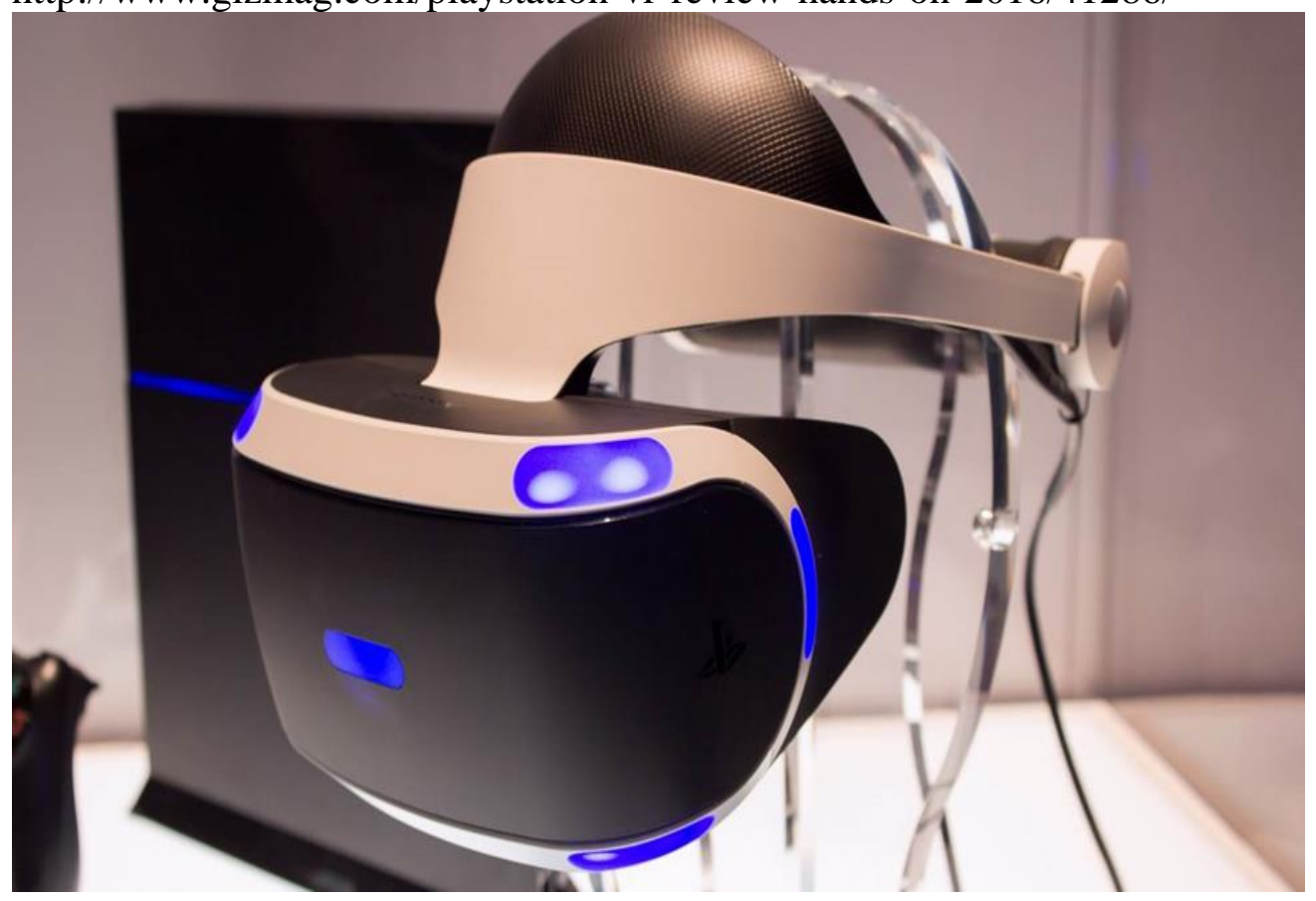


The price of admission will be under $\$ 500$ for this mind altering experience. This is a small fee considering how advance and revolutionary this product is. These factors lead experts to believe that introducing these technologies at an affordable price point will broaden the VR user base.

There will be more people involved in this virtual reality platform because the sell price is reasonable and not out of most consumers grasp. Social media which is a subcategory of entertainment will be well represented with Facebook's investment in the Oculus Rift [5].

Facebook which has a community of 2.2 billion worldwide social media users, has generated a lot of excitement and interest in this new piece of technology which it will be unveiled later this year. A lot of the excitement being focused on VR is thanks to Facebook investing billions of dollars in this technology which it views as a future technology sector it wants to be a leader of. With Facebook backing this technology platform it will bring confidence to hundreds of developers so that they can create unique content which will help illustrate the VR experience. Video camera makers are also preparing for the wave of films that will use VR technology.

A well-known cell phone company Nokia hopes to revitalize its brand by making high end VR video cameras. Nokia has experienced significant market share lost in the cell phone arena for the last decade, due to their phones suffering a decline in popularity to makers such as Apple. Nokia plans to lead the VR video movement and become a top competitor in this market. Nokia's Ozo VR camera as seen in [Figure 2], will bring a resurgence in Nokia's brand [6].

The Finnish company designed the camera which has the shape of a sphere and weighs about 10 pounds. It has eight $2 \mathrm{k}$ by $2 \mathrm{k}$ resolution cameras. Each lens is spaced out exactly as the distance between two human eyes. This feature enables it to have a realistic human view of all perspectives as the user moves around his head. The Ozo also has built in microphones in order to capture 3D audio.

The Ozo is unique in that users will not be viewing locations in a computer generated world. Instead they will be able to view locations in realtime anywhere around the world. Ozo wants to revolutionize VR and create a niche market for itself.

This technology will allow users to be in different places at any one time. This will expand on features available in existing video services such as Skype and bring a level of realism never before seen. A user will be able to experience the other caller's world on the other side of the line. The camera will retail for $\$ 50,000$ later this year. This is one example for how third party developers are utilizing up and coming VR technology and putting their own positive spin on it. 


\section{Conclusion}

As the research has mentioned earlier, VR technology has a positive impact on various areas of our economy but will have the greatest benefit in Health Care, Manufacturing, and Entertainment. These industries will see an entry of new and improved ways to approach not just services and production but also developing experience through virtual training.

VR technology is so revolutionary that it could be grown and create new supporting industries that exists because of its introduction in the mainstream this year by Facebook and Sony. In summary VR could enhance customer relations, employee training and organizational management. Most importantly it enriches our personal growth and helps us achieve a sense of ah and wonder that we once experienced so effortlessly as children.

\section{References:}

Carr, A (2014, January 16). Ford Taps Oculus Rift For Future Automobile Designs.

Retrieved from http://www.fastcompany.com/3024328/innovationagents/ford-taps-oculus-rift-for-future-automobile-designs

Darell, R. (2015, January). Is Virtual Reality The Next Big Thing In Entertainment?

Retrieved from http://www.bitrebels.com/entertainment/virtual-reality-nextthing-entertainment/

Fitzpatrick, A (2016, January 27). Exclusive: Google's New Head of Virtual Reality on What

They're Planning Next. Retrieved from http://time.com/4193755/googlecardboard-virtual-reality-clay-bavor-vr/

Gaudiosi, J. (2015, September 23). How Ford goes further with virtual reality. Retrieved

from http://fortune.com/2015/09/23/ford-virtual-reality/

Leather, A (2014, April). Para 1. Why Oculus Rift And Virtual Reality Could Be Huge And Not Just For Gamers, Forbes, April, 2014. Retrieved from http://www.forbes.com/sites/antonyleather/2014/04/14/why-oculus-riftand-virtual-reality-could-be-huge-and-not-just-for-gamers/\#212ca8df651f Maurizio, P (2015, November 28). Nokia's Ozo VR Camera Marks a Rebirth for the Phone Giant, Wired, November 28, 2015. Retrieved from http://www.wired.com/2015/11/nokia-ozo-vr-camera/

Shanklin, W. (2016, January 11). PlayStation VR hands-on: A more midranged alternative to the Rift and Vive, GiZmag, January 11, 2016. Retrieved from http://www.gizmag.com/playstation-vr-review-hands-on-2016/41286

Senson, A. (2016, January 6). Virtual Reality Therapy: Treating The Global Mental Health Crisis. Retrieved from 
http://techcrunch.com/2016/01/06/virtual-reality-therapy-treating-the-globalmental-health-crisis/

Silver, J., \& The Wachowski Brothers. (1999, March 31). The Matrix [Motion picture]. United States: Warner Bros.

Wiltz, C. (2013, July 17). The Oculus Rift - Changing Virtual Reality for Healthcare. Retrieved from http://www.mddionline.com/article/oculus-rift$\% \mathrm{E} 2 \% 80 \% 93$-changing-virtual-reality-healthcare 